\title{
Integrable Couplings: Generation, Hamiltonian Structures, Conservation Laws, and Applications
}

\author{
Huanhe Dong, ${ }^{1}$ Wen-Xiu Ma, ${ }^{2}$ Yufeng Zhang, ${ }^{3}$ and Tingting Chen ${ }^{1}$ \\ ${ }^{1}$ College of Mathematics and System Science, Shandong University of Science and Technology, Qingdao 266590, China \\ ${ }^{2}$ Department of Mathematics and Statistics, College of Arts and Sciences, University of South Florida, Tampa, FL, USA \\ ${ }^{3}$ College of Science, University of Mining and Technology, Xuzhou, China
}

Correspondence should be addressed to Huanhe Dong; donghuanhe@126.com

Received 6 November 2014; Accepted 6 November 2014; Published 24 December 2014

Copyright (C) 2014 Huanhe Dong et al. This is an open access article distributed under the Creative Commons Attribution License, which permits unrestricted use, distribution, and reproduction in any medium, provided the original work is properly cited.

Integrable couplings are coupled systems of integrable equations, which contain given integrable equations as their subsystems. Current topics include Hamiltonian structures, conservation laws, exact solutions, Darboux transformation, and symmetry constraints. Studies on integrable couplings explore new significant features and applications of integrable systems in various areas such as differential geometry, Lie algebras and groups, and differential equations of mathematical physics. During the last two decades, there has been a growing interest in integrable couplings and their theories in the community of mathematical physics. With the development of integrable systems, new theories and approaches in the area of integrable couplings constantly emerge. We think it is sufficiently important to gather and publish novel results on integrable couplings as a special issue. Of course, these papers placed in this special issue are not an exhaustive representation of the area of integrable couplings; they only show part of emerging results. It is our pleasure to share these interesting achievements with the readers who are interested in this area.

The special issue contains fifteen papers. Seven papers are related to integrable systems and their coupling systems and Hamiltonian structures. One paper discusses the semidirect sum of Lie algebras and its applications to integrable couplings. Three papers search for exact solutions of integrable equations. Two papers are devoted to studying the Rossby solitary wave which exists in ocean and atmosphere by applying integrable equations as well as their conservation laws. Finally, two papers cover some applications in other areas of mathematics and physics.

In a paper entitled "The semidirect sum of Lie algebras and its applications to $C-K d V$ hierarchy," $\mathrm{X}$. Dong et al. study integrable coupling of $\mathrm{C}-\mathrm{KdV}$ hierarchy and its biHamiltonian structures by Tu scheme and the quadratic-form identity with the help of semidirect sum of Lie algebras.

In a paper entitled "A new approach for generating the $T X$ hierarchy as well as its integrable couplings," G. Wang presents a loop algebra whose degrees are $2 \lambda$ and $2 \lambda+1$ to simply represent the above isospectral matrix and the TX hierarchy is derived. Specifically, through enlarging the loop algebra with 3 dimensions to 6 dimensions, a new integrable coupling of the TX hierarchy and its corresponding Hamiltonian structure are also obtained.

In a paper entitled "A complex integrable hierarchy and its Hamiltonian structure for integrable couplings of WKI soliton," $\mathrm{F}$. Yu et al. generate complex integrable couplings from zero curvature equations associated with matrix spectral problems. A direct application to the WKI spectral problem leads to a novel soliton equation hierarchy of integrable coupling system, and the Hamiltonian structure is also obtained. It is also indicated that the method of block matrix is an efficient and straightforward way to construct the integrable coupling system.

In a paper entitled "Some reduction and exact solutions of a higher-dimensional equation," G. Wang and Z. Han derived the conservation laws of the $(3+1)$-dimensional 
Zakharov-Kuznetsov equation by using Noether's theorem after an interesting substitution $u=v_{x}$ to the equation. Finally, some exact solutions of the Zakharov-Kuznetsov equation are constructed after solving the reduced equation.

In a paper entitled "Tri-integrable couplings of the Giachetti-Johnson soliton hierarchy as well as their Hamiltonian structure," L. Wang and Y.-N. Tang construct triintegrable couplings of the Giachetti-Johnson (GJ) hierarchy of soliton equations based on zero curvature equations from semidirect sums of Lie algebras and establish Hamiltonian structures of the resulting tri-integrable couplings by the variational identity.

In a paper entitled " $A$ few integrable couplings of some integrable systems and $(2+1)$-dimensional integrable hierarchies," B. Feng et al. obtain $(2+1)$-dimensional DS hierarchy by using the TAH scheme. Specifically, the integrable coupling of the DS hierarchy is derived.

In a paper entitled "Binary nonlinearization for AKNSKN coupling system," X. Wang et al. decompose the AKNSKN coupling system into two integrable Hamiltonian systems with the corresponding variables $x$ and $t_{n}$ under the bargmann symmetry constraint and the finite dimensional Hamiltonian systems are Liouville integrable.

In a paper entitled "Invariant solutions and conservation laws of the $(2+1)$-dimensional Boussinesq equation," W. Rui et al. investigate invariant solutions and conservation laws of the $(2+1)$-dimensional Boussinesq equation. The Lie symmetry approach is used to obtain the invariant solutions. Conservation laws for the underlying equation are derived by utilizing the new conservation theorem and the partial Lagrange approach.

In a paper entitled "Nonlinear integrable couplings of Levi hierarchy and WKI hierarchy," Z. Shan et al. discuss the nonlinear integrable couplings of the Levi hierarchy and the Wadati-Konno-Ichikawa (WKI) hierarchy by using the 8dimensional matrix Lie algebra.

In a paper entitled "Super-Hamiltonian structures and conservation laws of a new six-component super-AblowitzKaup-Newell-Segur hierarchy," F. You et al. propose a six-component super-Ablowitz-Kaup-Newell-Segur (AKNS) hierarchy by the zero curvature equation associated with Lie superalgebras. Supertrace identity is used to furnish the super-Hamiltonian structures for the resulting nonlinear superintegrable hierarchy.

In a paper entitled "Conservation laws and self-consistent sources for an integrable lattice hierarchy associated with a three-by-three discrete matrix spectral problem," Y.-Q. Li and B.-S. Yin deduce a lattice hierarchy with self-consistent sources from a three-by-three discrete matrix spectral problem.

In a paper entitled "Benjamin-Ono-Burgers-MKdV equation for algebraic Rossby solitary waves in stratified fluids and conservation laws," $\mathrm{H}$. Yang et al. derive the BenjaminOno-Burgers-MKdV (BO-B-MKdV) equation which governs algebraic Rossby solitary waves in stratified fluids. By analysis and calculation, some conservation laws are derived from the BO-B-MKdV equation.

In a paper entitled "Dissipative nonlinear Schrödinger equation for envelope solitary Rossby waves with dissipation effect in stratified fluids and its solution," Y. Shi et al. solve the so-called dissipative nonlinear Schrödinger equation.

\section{Acknowledgments}

We would like to thank the authors for their excellent contributions and patience in assisting us. Finally, the fundamental work of all the reviewers of these papers is also very warmly acknowledged.
Huanhe Dong

Wen-Xiu Ma

Yufeng Zhang

Tingting Chen 